\title{
Spectroscopic investigations of the lowest triplet state of $s$-tetrazines
}

\author{
F. Gückel ${ }^{a, 1}$, A.H. Maki ${ }^{b, 2}$, F.A. Neugebauer ${ }^{a}$, D. Schweitzer ${ }^{b}$ and H. Vogler ${ }^{c}$ \\ a Max-Planck-Institut für medizinische Forschung, Jahn Strasse 29, 69 Heidelberg, Germany \\ b 3. Physik. Institut, Universität Stuttgart, Pfaffenwaldring 57, 7 Stuttgart 80, Germany \\ c Siemens AG, Otto-Hahn-Ring 6, 8 Munich 83, Germany
}

Received 3 February 1992

\begin{abstract}
We report on the electronic and triplet state ODMR spectroscopy of s-tetrazıne, 3,6-dimethyl-, 3,6-dimethoxy- and 3,6bis (methylthio)-s-tetrazines. Zero field splitting parameters were obtained by ODMR monitoring both the delayed fluorescence and the phosphorescence in neat single crystals. The $S_{1}$ and $T_{1}$ exciton electronic origins are reported. The zero field splitting tensor is nearly axially symmetric with $|D|$ approximately $0.4 \mathrm{~cm}^{-1}$. This value is found to be in good agreement with calculations based on magnetic dipole-dipole coupling in a ${ }^{3} B_{3 u}\left(n \pi^{*}\right)$ state. The calculations predict a positive value for $D$ with the major axis passing through the $C$ atoms at the 3 and 6 positions.
\end{abstract}

\section{Introduction}

A great deal of interest has been devoted to the electronic spectroscopy and photochemistry of $s$-tetrazine (1,2,4,5-tetraazabenzene, 1$)$. Early studies of the vapor phase absorption spectra [1-5] revealed two clear absorption band systems; an intense system near $250 \mathrm{~nm}$ was assigned to the analogue of the ${ }^{1} B_{2 u} \leftarrow{ }^{1} A_{g}\left(\pi-\pi^{*}\right)$ transition of benzene, while bands of a weaker system near $550 \mathrm{~nm}$ have been shown to belong to a ' $B_{3 u} \leftarrow^{1} A_{B}\left(n-\pi^{*}\right)$ transition $\# 1$. A broad absorption at $\approx 350 \mathrm{~nm}$ has been assigned [3] as a second $\left(n-\pi^{*}\right)$ transition. Fluorescence of 1 $\left({ }^{\prime} B_{3 u} \rightarrow{ }^{1} A_{g}\right.$ ) was observed by Chowdhury and Goodman in a glass at $77 \mathrm{~K} \mathrm{[7],} \mathrm{who} \mathrm{also} \mathrm{noted} \mathrm{the} \mathrm{ab-}$ sence of any detectable phosphorescence accompa-

1 Now at: Institut für Klınısche Radiologie, Klinikum Mannheim, Mannheim, Germany.

2 On leave from the Chemistry Department of the University of California, Davis, USA.

\#T The symmetry designations are based on the molecular axis system used in the early spectroscopic work [2-5], i.e., the $z$-axis goes through the $C_{3}$ and $C_{6}$ positions of $s$-tetrazine and the $x$-axis is normal to the molecular plane. A more recent axis system convention (see, for instance ref. [6]) is based on the transformation, $(x, y, z) \rightarrow(y, z, x)$, in which case the excited state designations would be ${ }^{1} B_{1 n}\left(\pi-\pi^{*}\right)$ and ${ }^{1} B_{2 u}\left(n-\pi^{*}\right)$. In this paper, we retain the earlier axis convention. nying excitation in the singlet manifold. The weak ${ }^{3} B_{3 u} \leftarrow{ }^{1} A_{g}$ system of 1 was identified by Livak and Innes [8] in the vapor phase absorption spectrum; the corresponding phosphorescence was observed by McDonald and Brus [9] following direct excitation in the $T_{1} \leftarrow S_{0}$ system. These authors also noted the apparent lack of efficient $S_{1} \rightarrow T_{1}$ intersystem crossing (isc) in 1. Single crystal absorption spectra of 1 [10] and the related compound 3,6-dimethyl-s-tetrazine, $2[11]$, yielded ${ }^{3} B_{3 u} \leftarrow{ }^{1} A_{g}$ band systems in absorption, and phosphorescence was observed upon excitation in this band. The lack of perceptible isc in 1 was also pointed out by Hochstrasser and King [10]. The phosphorescence lifetimes in the gas phase $\tau=72 \pm 2 \mu \mathrm{s}[9]$, and in the neat crystal at $\leqslant 4.2 \mathrm{~K}$, $\tau=96.8 \pm 2.1 \mu \mathrm{s}[10]$ are similar. Their magnitude implies the presence of efficient radiationless deactivation.

Vemulapalli and Cassen [12] reported an extremely low vapor phase fluorescence quantum yield for 1 , implying efficient radiationless deactivation of ${ }^{1} B_{3 u}$ even in the absence of a perceptible isc.

Direct fluorescence lifetime measurements of 1 in the gas phase [13] and in a seeded supersonic jet free expansion [14] by ps-pulse methods have given a lifetime of $\tau_{\mathrm{F}} \approx 800 \mathrm{ps}$. From the integrated gas phase oscillator strength of the ${ }^{1} B_{3 u} \leftarrow^{1} A_{g}$ transition of 
$5.4 \times 10^{-3}[9,12]$ one can estimate the fluorescence quantum yield of $\Phi_{\mathrm{F}} \approx 0.9 \times 10^{-3}$. The efficient photochemical decomposition of 1 in both the low temperature solid state and in the gas phase was pointed out by Meyling et al. [15] who reported that the gas phase photodissociation quantum yields for $1-h_{2}, 1$ $d_{2}$ and 2 have the value of $1.3 \pm 0.3$ with optical pumping of ${ }^{1} \mathrm{~B}_{3 \mathrm{u}} \leftarrow^{1} \mathrm{~A}_{\mathrm{g}}$. This conclusion has been disputed in the case of $\mathbf{2}$ by Hochstrasser and co-workers [16], who find a significantly lower value. The observation of $\mathrm{HCN}$ and $\mathrm{N}_{2}$ in the photoproducts of 1 by Hochstrasser and co-workers upon photolysis in the solid state at $1.6 \mathrm{~K}$ [17-19] established that the principal photoreaction is

1

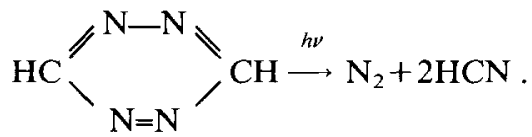

Photoselective decomposition of 1 using an $\mathrm{Ar}^{+}$. pumped dye laser was utilized to produce isotopically enriched $\left({ }^{15} \mathrm{~N},{ }^{13} \mathrm{C},{ }^{2} \mathrm{D}\right)$ photoproducts in the solid state at $T \leqslant 4.2 \mathrm{~K}$ [17-19]. It is thus apparent that the low $\Phi_{\mathrm{F}}$ and inefficient isc may be connected with the efficient photochemical decomposition of 1 . Although the room temperature photochemical decomposition of 1 in hexane solution occurs by a single photon process upon $S_{1} \leftarrow S_{0}$ excitation, the photoreaction of 1 and $\mathbf{2}$ in molecular crystals at low temperature has been shown to occur by a process involving the absorption of two photons [20]. It is of interest that although $\mathbf{1}$ apparently is photolyzed with about unit quantum yield under $S_{1} \leftarrow S_{0}$ excitation in the gas phase [15], 2 is found at room temperature to be at least 100 times as resistant to photolysis in $n$ hexane solution as $\mathbf{1}$ which decomposes efficiently under these conditions [20]. However, both 1 and 2 decompose at room temperature in hexane when excited using $\lambda<280 \mathrm{~nm}$. These results indicate that the photochemistry of 1 and $\mathbf{2}$ differ considerably and that the solid state low temperature photochemistry differs considerably from that which occurs in hexane solution at room temperature. Recent seeded supersonic free jet expansion measurements by Levy and co-workers have shown that the quantum yield for $s$ tetrazine photolysis varies with both the 3,6-substituents and the vibronic band involved in the ${ }^{1} B_{3 u} \leftarrow{ }^{1} A_{g}$ excitation [21]. Their work shows that methyl sub- stitution and amine substitution in particular leads to significant increases in $\Phi_{\mathrm{F}}$ and $\tau_{\mathrm{F}}$ as well as decreases in photolysis quantum yields. Also, the photolysis quantum yield is found to increase monotonically with excess vibrational energy in the excited state. These conclusions were also reached from gas phase photolysis quantum yield measurements on 2 [16]. The enhanced stability of 3,6-substituted $s$-tetrazines against photolysis also has been observed for 3,6-diphenyl-s-tetrazine [22].

The gas phase photochemistry of 1 has been investigated recently by Lee and co-workers [23] using the method of photofragment-translational spectroscopy. They find that 1 reverts to a highly vibrationally excited ground electronic state by internal conversion following either ${ }^{1} B_{3 u} \leftarrow{ }^{1} A_{g}(0-0$ at 18128 $\left.\mathrm{cm}^{-1}\left[{ }^{9}\right]\right)$ or ${ }^{1} B_{2 u} \leftarrow{ }^{1} A_{g}(280 \mathrm{~nm})$ excitation, and then converts into $2 \mathrm{HCN}+\mathrm{N}_{2}$ via concerted triple dissociation. The transition state on the ${ }^{1} A_{g}$ potential energy surface which leads to the triple dissociation has a high energy, thereby producing a very repulsive reaction coordinate with most of the available energy going into translational kinetic energy of the photofragmented products. According to this interpretation $[23], \tau_{\mathrm{F}}$ is determined by the internal conversion process. Calculations of the ${ }^{1} A_{s}$ potential energy surface of 1 [ 24] place the transition state leading to the triple dissociation at $\approx 16450 \mathrm{~cm}^{-1}$ above the zero point which suggests that isc from the ${ }^{3} B_{3 u}(0-0$ at $13608 \mathrm{~cm}^{-1}$ [9]) would produce ${ }^{1} \mathrm{~A}_{\mathrm{g}}$ with insufficient energy to enter the reaction channel utilized when ${ }^{1} \mathrm{~B}_{3 \mathrm{u}}$ is excited. To our knowledge, there have been no conclusive experimental reports of photolysis of either 1 or 2 occurring via the triplet state.

Most of the work on 1 and its 3,6-derivatives thus has focused on the low-lying singlet states and the associated high resolution spectroscopy, photophysics, and photochemistry. We report in this paper on low temperature spectroscopic measurements, particularly by optical detection of magnetic resonance (ODMR) on the properties of the ${ }^{3} B_{3 u}$ state of 1,2 , 3,6-dimethoxy-s-tetrazine (3) and 3,6-bis (methylthio )-s-tetrazine (4), in neat single crystals. The mode of excitation for 1 and 2 was directly by the ${ }^{3} B_{3 u} \leftarrow{ }^{1} A_{g}$ transition for the reasons that are apparent from the discussion above. However, for 3 and $\mathbf{4}$ we were able to populate the ${ }^{3} B_{34}$ state by optical pumping of ${ }^{1} B_{3 u} \leftarrow{ }^{1} A_{g}$ and to make measurements in dilute ma- 
trices as well, demonstrating that isc processes in these crystals occur efficiently, probably because of reduced rates of internal conversion and photofragmentation of the ${ }^{1} B_{3 u}$ state. Of particular interest to us was measurement of the zero field splittings ( $\mathrm{zfs}$ ) of the ${ }^{3} B_{3 u}, n \pi^{*}$ state. These are predicted to be considerably larger than those found in $\pi \pi^{*}$ states of analogous aromatic molecules because of the large effects of the $\mathrm{N}$ atoms with their one-center contributions to the magnetic dipole-dipole interaction [25].

\section{Experimental}

Measurements were made, in general, on neat single crystals of 1-4. Their synthesis has been reported previously [26]. The compounds were purificd using chromatography, and pure single crystals were obtained by sublimation in the dark under high vacuum.

The samples were optically excited in the ${ }^{3} B_{3 u} \leftarrow{ }^{1} A_{g}$ absorption system, or in ${ }^{1} B_{3 u} \leftarrow{ }^{1} A_{g}$. In each case a tunable dye laser (Coherent, model 590; output power, $10-40 \mathrm{~mW}$ ) was pumped by a Coherent model $3000 \mathrm{~K}$ krypton ion laser. The bandwidth of the dye laser output was $\approx 0.5 \mathrm{~cm}^{-1}$. Oxazine- 1 was used for optical pumping in the $T_{1} \leftarrow S_{0}$ region, while rhodamine $6 \mathrm{G}$ was used for pumping the $S_{1} \leftarrow S_{0}$ transition. The samples were optically pumped in a liquid $\mathrm{He}$ bath maintained at $T=1.3 \mathrm{~K}$.

The position of the $T_{1}$ exciton band was obtained by monitoring singlet $x$-trap delayed fluorescence while sweeping the dye laser frequency through the ${ }^{3} B_{3 u} \leftarrow{ }^{1} A_{g}$ origin. The $S_{1}$ exciton band origin could be identified by its intensity which is several orders of magnitude larger than $x$-trap absorption.

The OMDR apparatus has been described previously [27]. Signal averaging of the phosphorescence or delayed fluorescence was carried out using a transient digitizer (Biomation 8100) interfaced with either a computer system (Dietz Mincal 621) or a computer of average transients (Princeton Applied Research, model 4202). The microwave power was amplified up to $8 \mathrm{~W}$ using several TWT amplifiers. The zfs parameters were obtained from the microwave-induced slow-passage optical responses in zero applied magnetic field [28]. The short lifetimes of the triplet sublevels, $\tau \leqslant 100 \mu$ s precluded the mea- surements of the triplet sublevel kinetic parameters by conventional ODMR methods using the existing equipment.

\section{Results}

\subsection{The emission spectra of the s-tetrazines}

The emission spectra of s-tetrazines 1 through 4 with $S_{1} \leftarrow S_{0}$ excitation are shown in the lower parts of figs. $1-4$, respectively. The crystal ${ }^{1} B_{3 u} \leftarrow^{1} A_{g}$ origin of 1 occurs at $18414 \pm 3 \mathrm{~cm}^{-1}$, and the fluorescence consists of a dominant progression of groups of sharp $\mathrm{x}$-trap emission lines overlapping with phonon side bands separated by $740 \mathrm{~cm}^{-1}$ (fig. 1) in agreement with previous observations [10]. Excitation of the $\mathrm{S}_{1} \leftarrow \mathrm{S}_{0}$ band produces no detectable phosphorescence in single crystals of 1 but in 3 , and increasingly in 4 , clear phosphorescence spectra are found, indi-

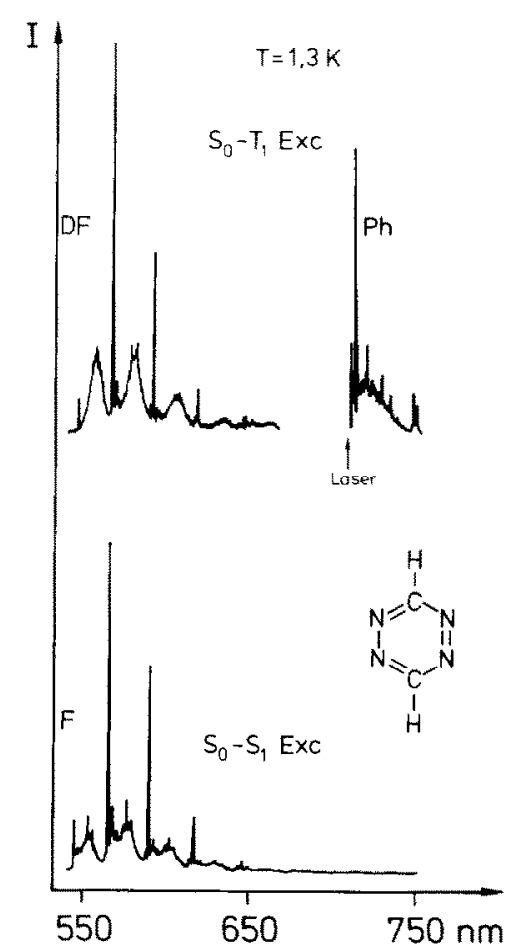

Fig. 1. Emission spectra of s-tetrazine (1), lower part with $S_{1} \leftarrow S_{0}$ excitation and upper part with direct laser excitation in the triplet exciton band ( $F=$ fuorescence, $P=$ phosphorescence, $\mathrm{DF}=$ delayed fluorescence). 


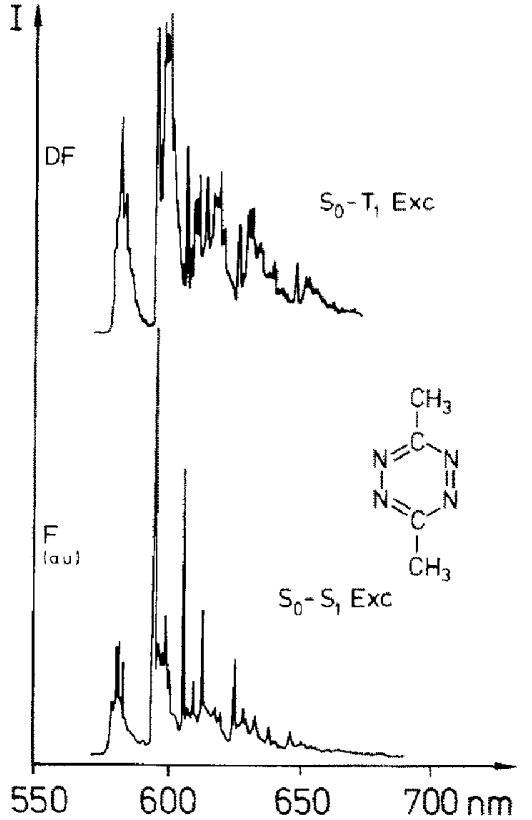

Fig. 2. Emission spectra of 3,6-dimethyl-s-tetrazine (2), lower part with $S_{1} \leftarrow S_{0}$ excitation and upper part with direct laser excltation in the triplet exciton band ( $F=$ fluorescence, $D F=$ delayed fluorescence).

cating significant isc in these crystals (figs, 3 and 4 ). We were not able to determine whether or not detectable phosphorescence occurs in $\mathbf{2}$, since this phosphorescence would be found beyond the $750 \mathrm{~nm}$ longwavelength limit of our monochromator. Previous measurements on 2 , however, have shown that in the condensed phase at low temperature, the isc quantum yield in less than $10^{-4}$ [29].

Upon direct laser excitation in the triplet exciton band, each of the crystals emits phosphorescence as well as intense delayed fluorescence (upper section of figs. 1-4). The delayed fluorescence contains the same bands as the directly excited fluorescence, but relatively greater emission intensity originates from deep $x$-traps. This is consistent with the origin of the delayed fluorescence mainly from the heterofusion of triplet excitons and triplet $x$-traps [30].

In fig. 5, we compare the phosphorescence spectra of 3 produced by $S_{1}$ (band) $\leftarrow S_{0}, T_{1}$ (band) $\leftarrow S_{0}$ and $T_{1}$ (trap) $\leftarrow S_{0}$ excitation. These excitations were carried out at 18382,14292 and $14206 \mathrm{~cm}^{-1}$, respectively. The wavelength of the laser excitation in the latter case is shown by an arrow on the lowest tracing

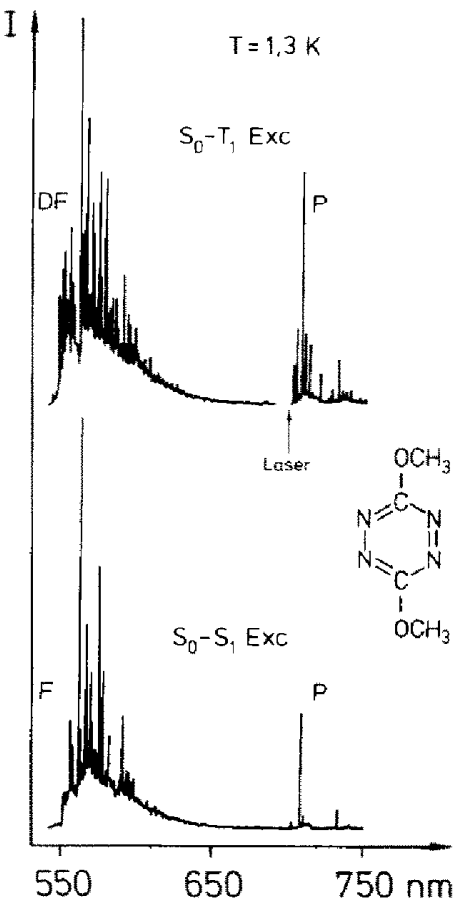

Fig. 3. Emission spectra of 3,6-dimethoxy-s-tetrazine (3), lower part with $S_{1} \leftarrow S_{0}$ excitation and upper part with direct laser excitation in the triplet exciton band ( $F=$ fluorescence, $P$ - phosphorescence, $D F=$ delayed fluorescence $)$.

in fig. 5. The number of emission lines is considerably reduced when a single $x$-trap is optically pumped. Comparison of the two upper spectra of fig. 5 shows that the population of emitting traps depends also on whether the $S_{1}$ or $T_{1}$ band is optically pumped. Since delayed fluorescence is observed only in connection with excitation to the $T_{1}$ band and not to the $T_{1} x$ trap, it can be conluded that it arises from triplettriplet annihilation rather than a two-photon excitation process. The $T_{1}$ band thus could be located unambiguously by sweeping the dye laser wavelength in the region of the $T_{1}$ origin while monitoring the delayed fluorescence. The delayed fluorescence excitation spectra taken at the $T_{1}$ band origin of the $s$-tetrazines 1, 2 and 4 are shown in fig. 6 . The corresponding spectrum of 3 (fig. 7) exhibits a four-component fine structure with splittings ranging between 1.5 and 6.0 $\mathrm{cm}^{-1}$. The relative intensity of the lines varies with crystal orientation in the laser beam. Attempts at photochemical hole-burning revealed that the structure does not arise from individual sites. The struc- 


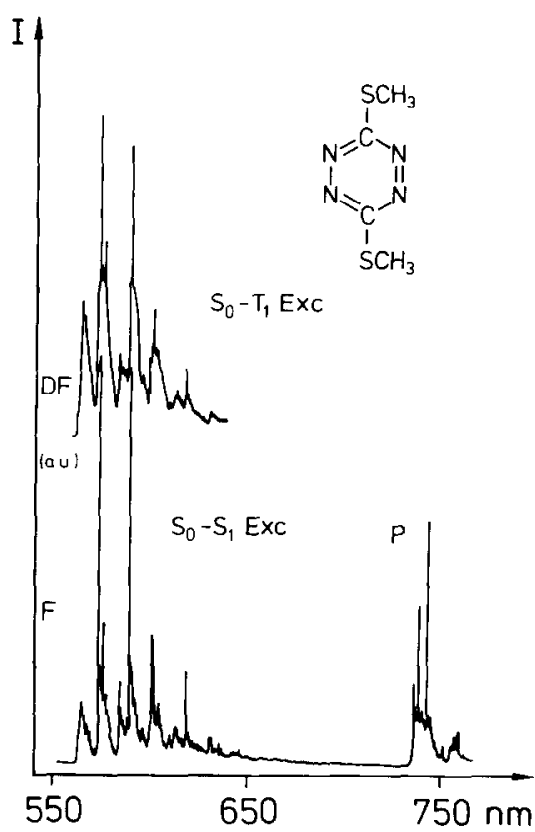

Fig. 4. Emission spectra of 3,6-bis (methylthio)-s-tetrazine (4), lower part with $S_{1} \leftarrow S_{0}$ excitation and upper part with direct laser excitation in the triplet exciton band $(F=$ fluorescence, $\mathrm{P}=$ phosphorescence, $\mathrm{DF}=$ delayed fluorescence $)$.

turc probably is duc to Davydov splittings of the four inequivalent molecules in the unit cell of the orthorhombic crystal, space group $\mathrm{D}_{2 \mathrm{~h}}^{15}[31,32]$. The energies of the $T_{1}$ and $S_{1}$ exciton bands of the neat crystals are given in table 1 . Table 1 also includes the fluorescence and phosphorescence origins of 3 and 4 which were obtained in a dilute $n$-octane Shpolskii matrix at $1.3 \mathrm{~K}$.

\subsection{Optically detected magnetic resonance}

The triplet state was excited in general by optical pumping of the crystal $T_{1}$ origin in order to minimize photolytic damage of the crystal. The estimated rate of decomposition of 1 through $S_{1}$ excitation is a factor of $10^{5}$ larger than through $T_{1}$ excitation [17]. Since all the $s$-tetrazines emit delayed fluorescence, the ODMR signals were sought by monitoring both a particular $T_{1}$ trap emission line (except for 2 ) and a delayed fluorescence line. Fig. 8a shows two narrow ODMR lines of 1 which were detected by monitoring a triplet trap emission line at $14065 \mathrm{~cm}^{-1}$ which lies

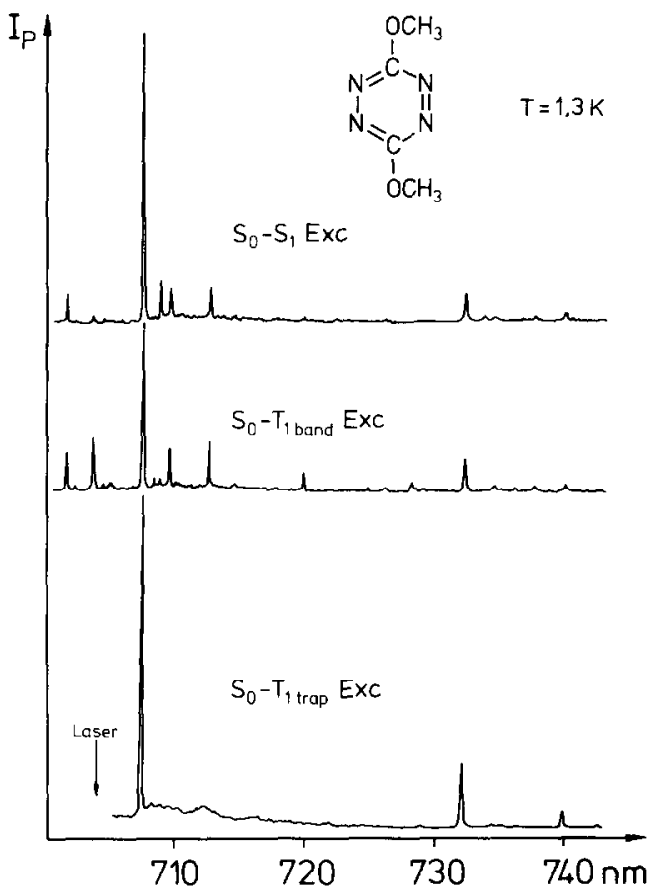

Fig. 5. Phosphorescence spectra of 3,6-dimethoxy-s-tetrazine (3) obtained by different excitation modes (see text).

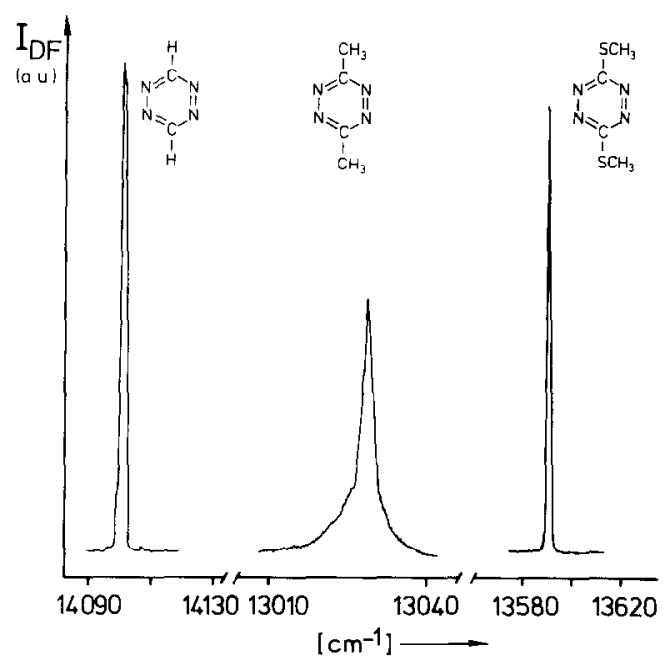

Fig. 6. Excitation spectra of the triplet $T_{1}$ exciton and band origins of (a) s-tetrazine 1, (b) 3,6-dimethyl-s-tetrazine 2 and (c) 3,6bis (methylthio)-s-tetrazine 4. 


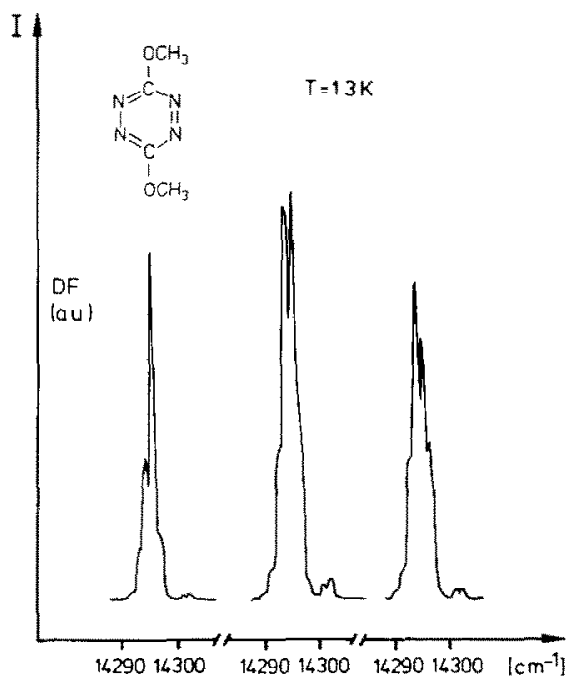

Fig. 7. Excitation spectra of the triplet $T_{1}$ exciton band origin of 3,6-dimethoxy-s-tetrazine (3) at three different crystal orientations with respect to the polarization vector of the exciting laser light.

$44.5 \mathrm{~cm}^{-1}$ below the triplet exciton band (table 1 ). The peak frequencies and linewidths are given in table 2. A third signal could not be found, but by analogy with the ODMR spectra of the other s-tetrazines (see below) it would occur at the difference of the two observed frequencies. No corresponding ODMR signals were found monitoring the delayed fluorescence. Adopting the convention $|D| \geqslant 3|E|$, the sig- nals are assigned as $|D|+|E|$ and $|D|-|E|$. Conditions for observing these ODMR signals (as well as the others reported below) are rather stringent. High microwave power, generally exceeding $1 \mathrm{~W}$, is required for saturation of sublevel populations due to their short decay lifetimes, $<100 \mu$ s, while relatively low optical excitation intensities $(\approx 10 \mathrm{~mW})$ are also necessary to maintain a sufficently low triplet exciton concentration in order that rapid heterofusion processes do not eliminate the sublevel population differences which are necessary in order to observe the ODMR signals [31]. The zero field ODMR transitions of 2 , detected by monitoring a delayed fluorescence line at $17042.5 \mathrm{~cm}^{-1}$, are given in fig. $8 \mathrm{~b}$. The $|D|-|E|$ signal could not be detected directly, but was observed using EEDOR, with continuous excitation of the high frequency transition. The ODMR signals of $\mathbf{3}$ and $\mathbf{4}$, in which phosphorescence detection via a selected trap are compared with the delayed fluorescence-detected ODMR are shown in figs. $8 \mathrm{c}$ and $8 \mathrm{~d}$, respectively. All the ODMR data are collected in table 2 , including phosphorescence-detected ODMR signals of 3 and 4 observed in a $n$-octane matrix. Several individual triplet $x$-traps of 3 and 4 could be identified by their differing $z \mathrm{fs}$. The phosphorescence-detected $x$-trap signals are generally quite narrow structureless lines. The corresponding delayed fluorescence-detected signals are broader and contain partially resolved structures. Signals detected in the phosphorescence have a polarity oppo-

Table 1

Band origins of the $S_{1}$ and $T_{1}$ excited states (errors $\pm 3 \mathrm{~cm}^{-1}$ ) of neat crystals of $s$-tetrazine (1), 3,6-dimethyl-s-tetrazine (2), 3,6dimethoxy-s-tetrazıne (3) and 3,6-bis (methylthio)-s-tetrazine (4) as well as $S_{1}$ and $T_{1}$ of 3 and 4 in $n$-octane

\begin{tabular}{|c|c|c|c|c|}
\hline & \multicolumn{2}{|c|}{ Exciton band } & \multicolumn{2}{|l|}{ X-Trap } \\
\hline & $\mathrm{S}_{1}\left(\mathrm{~cm}^{-1}\right)$ & $T_{1}\left(\mathrm{~cm}^{-3}\right)$ & $S_{1}\left(\mathrm{~cm}^{-1}\right)$ & $T_{1}\left(\mathrm{~cm}^{-1}\right)$ \\
\hline 1 & 18414 & 14100.5 & & 14075 \\
\hline 2 & 17352 & 13029 & & \\
\hline \multirow[t]{4}{*}{3} & 18382 & 14293 & & 14253 \\
\hline & & 14294.5 & & \\
\hline & & 14300.5 & & \\
\hline & & 14302 & & \\
\hline 4 & 17794 & 13591 & & 13576 \\
\hline 3 in $n$-octane & & & 18348 & 13746 \\
\hline 4 in $n$-octane & & & 17953 & 13363 \\
\hline
\end{tabular}



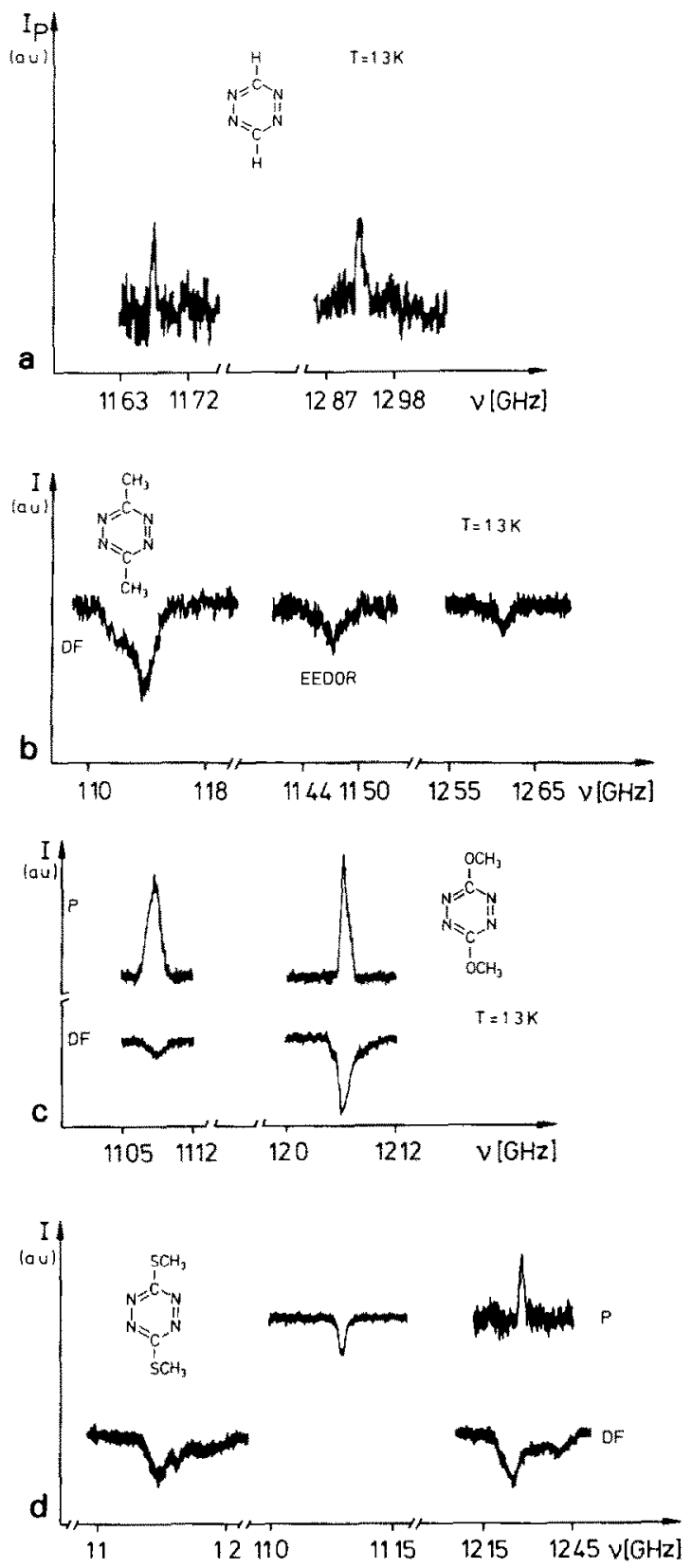

Fig. 8. ODMR signals of (a) s-tetrazine 1, (b) 3,6-dimethyl-stetrazine 2, (c) 3,6-dimethoxy-s-tetrazine 3 and (d) 3,6bis (methylthio)-s-tetrazine 4 (see text).

site to the corresponding delayed fluorescence-detected signals. Bccause of the close agreement between the ODMR frequencies of 3 and 4 observed in $n$-oc- tane and those in the crystal, we can be quite confident that the triplet $x$-traps and not triplet excitons are being measured and that they are minimally distorted individual $s$-tetrazine molecules. The observed $\mathrm{zfs}$ therefore represent properties of the individual molecules, and can be discussed on that basis.

The triplet state transitions observed by the delayed fluorescence responses must occur in the triplet $\mathrm{x}$-traps, their effect being transferred by altered rates of the heterofusion process. In the case of $\mathbf{3}$ and $\mathbf{4}$ for which both delayed fluorescence and $x$-trap phosphorescence were compared, we always found a close correspondence between the $\mathrm{zfs}$ of the major peak in the structured delayed fluorescence detected signal and that of one of the individually monitored $x$-traps (see table 2).

\section{Discussion}

Since the molecular axes corresponding to the zero field magnetic sublevels were not determined experimentally, we must rely on a theoretical calculation to suggest their appropriate ordering. Qualitatively, the magnitude of $|D|$ in $1-4\left(\approx 0.4 \mathrm{~cm}^{-1}\right)$ determined in this work is significantly larger than the experimental value [33] for benzene, a typical $\pi \pi^{*}$ triplet state in an isoelectric and isostructural molecule $\left(0.158 \mathrm{~cm}^{-1}\right)$; this suggests at the outset that the designation of the lowest triplet state as $n \pi^{*}$ is correct. Furthermore, the lowest ${ }^{3} B_{3 u}$ state of s-tetrazine is well separated energetically $(>1 \mathrm{eV})$ from the lowest triplet $\pi \pi^{*}$ excited state that can interact by spin-orbit coupling thus influencing the zfs [34]. Moreover, spin-orbit coupling with the $S_{1}\left({ }^{1} B_{3 u}\right)$ state is forbidden by symmetry rules, as well as spin-orbit coupling with the dominant vibrational mode, the totally symmetric $\nu_{6 a}^{\prime}$ [10]. Thus, a magnetic dipole-dipole model for the zfs in s-tetrazine seems appropriate.

The size of $|D|$ in pyrazine, $0.33 \mathrm{~cm}^{-1}[35,36]$, is in accord with the large value expected for magnetic dipole-dipole coupling in a $n \pi^{*}$ state from one-center terms of $\mathrm{N}$ atoms containing large spin density in both $\mathrm{n}$ and $\pi$ orbitals [25], although this value may be reduced somewhat by a contribution to the $\mathrm{zfs}$ from spin-orbit coupling with an energetically proximal ${ }^{3} \pi \pi^{*}$ state [37].

The theoretical scheme we have used for the cal- 
Table 2

ODMR triplet zero field splitting transitions $D+|E|$ and $D-|E|(\mathrm{GHz})$, ODMR linewidth (lw, in $\mathrm{MHz}$ ) and detection wavelengths ( $\AA ; \mathrm{P}=$ phosphorescence, $\mathrm{DF}=$ delayed fluorescence) of neat crystals of $s$-tetrazine (1), 3,6-dimethyl-s-tetrazine (2), 3,6-dimethoxy-stetrazine (3) and 3,6-bis (methylthio)-s-tetrazine (4) as well as of 3 and 4 in $n$-octane

\begin{tabular}{|c|c|c|c|c|c|c|}
\hline & $D+|E|$ & lw & $D-|E|$ & lw & $\begin{array}{l}\text { Detection } \\
\text { waveleng }\end{array}$ & \\
\hline 1 & 12.926 & 7.5 & 11.675 & 6 & 7114.5 & $\mathbf{P}$ \\
\hline 2 & 12.616 & 10 & 11.478 & 14 & 5867.7 & DF \\
\hline 3 & 12.061 & 15 & 11.085 & 15 & 5614.3 & Dr \\
\hline trap 1 & 12.046 & 9 & 11.101 & 8 & 7014.5 & $\mathbf{P}$ \\
\hline trap 2 & 12.086 & 10 & 11.125 & 15 & 7035.5 & $\mathbf{P}$ \\
\hline trap 3 & 12.061 & 10 & 11.081 & 15 & 7071.5 & $\mathbf{P}$ \\
\hline $\operatorname{trap} 4$ & 12.048 & 11 & 11.116 & 14 & 7121 & $\mathrm{P}$ \\
\hline in $n$-octane & 12.232 & 9 & 11.185 & 10 & 7274.5 & $P$ \\
\hline 4 & 12.232 & 20 & $2|E|=1.147$ & 15 & 5728 & $\mathrm{DF}$ \\
\hline trap 1 & 12.238 & 7.5 & 11.087 & 9 & 7366 & $\mathrm{P}$ \\
\hline trap 2 & 12.237 & 12 & 11.170 & 10 & 7390 & $\mathbf{P}$ \\
\hline trap 3 & 12.288 & & 11.127 & & 7394 & $\mathbf{p}$ \\
\hline trap 4 & 12.261 & & 11.116 & & 7432 & $\mathrm{P}$ \\
\hline in $n$-octane & 12.475 & 8 & 11.246 & 10 & 7483.5 & $\mathrm{p}$ \\
\hline
\end{tabular}

culation of the contributions to $D$ and $E$ from dipolar coupling in aza-aromatic molecules has been published previously [38] and will not be outlined here. The calculations for s-tetrazine [38] have been extended in this paper to include 3,6-dimethyl-s-tetrazine 2 and 3,6-dihydroxy-s-tetrazine 3a, an analog of 3. The calculated values are compared with our measured values in table 3 . Also included are results for the similar molecule, pyrazine. The calculations verify the dominance of the one-center terms in the dipole-dipole interactions. These terms also determine the direction of the $\bar{z}$-axis, which is the principal magnetic axis defining the minimum separation of the spin pair. With this definition, $T_{\bar{z}}$ will be the lowest energy sublevel with energy $\epsilon_{\bar{z}}=-\frac{2}{3} D$. For 1 and $3 a$, the $\bar{z}$-axis is calculated to run through the $\mathrm{C}$ atoms at positions 3 and 6 , while for pyrazine, the $\bar{z}$-axis is in the molecular plane, but normal to the line drawn between the $\mathrm{N}$ atoms, in agreement with Zeeman effect studies [39], and magnetophotoselection measurements [35]. The calculated values of $D$ for 1-3 are about $12 \%$ larger than the experimental ones, al-

Table 3

Calculated and experimental triplet zero field splitting parameters $|D|$ and $|E|$ (values given in $\mathrm{cm}^{-1}$ ) of $s$-tetrazine (1), 3,6-dimethyls-tetrazine (2), 3,6-dimethoxy-s-tetrazine (3), 3,6-bis (methylthio)-s-tetrazine (4) and pyrazine ${ }^{\text {a) }}$

\begin{tabular}{|c|c|c|c|c|c|c|}
\hline & \multicolumn{4}{|c|}{ Calculated } & \multicolumn{2}{|c|}{ Experimental } \\
\hline & $D$ & $E$ & $D_{1}^{\text {b) }}$ & $D_{2}^{\text {b) }}$ & $|D|$ & $|E|$ \\
\hline 1 & 0.461 & 0.049 & 0.481 & -0.019 & 0.410 & 0.021 \\
\hline 2 & 0.441 & 0.048 & 0.469 & -0.028 & 0.402 & 0.019 \\
\hline 3 & 0.438 & 0.045 & 0.459 & -0.021 & 0.386 & 0.016 \\
\hline 4 & - & - & - & - & 0.390 & 0.019 \\
\hline pyrazine & 0.467 & 0.040 & 0.481 & -0.014 & $0.331^{\text {a }}$ & $0.007^{\text {a) }}$ \\
\hline
\end{tabular}

*) Data from refs. [35] and [36].

b) One-center and two-center contributions to $D$, respectively. 
though they follow the variation among these $s$-tetrazines reasonably well. We can thus feel reasonably confident that $D$ is positive, as it is for pyrazine [39]. The magnitude of $E$ is overestimated in the calculation by about a factor of two and thus we cannot confidently give it a sign. The calculated $D$ and $E$ values of pyrazine are much less accurate, $D$ being overestimated by some $40 \%$, suggesting that spin-orbit contributions to the zfs are important in this molecule. Spin-orbit coupling with a nearby ${ }^{3} \mathrm{~B}_{1 \mathrm{u}}\left(\pi-\pi^{*}\right)$ state lying $\approx 1600 \mathrm{~cm}^{-1}$ above the lowest ${ }^{3} B_{3 u}\left(n-\pi^{*}\right)$ state of pyrazine, as suggested by the work of Hochstrasser and Marzzacco [37] would have the effect of reducing the size of $D$.

Unfortunately, we were unable to measure directly the kinetic and radiative parameters of the individual triplet sublevels. This would have made it possible to obtain direct information about the triplettriplet annihilation processes which arise from interactions between $\mathrm{x}$-traps and triplet excitons. Some speculations can be made however, which appear to account qualitatively for what we are able to measure. The important observations are: (a) that the intensity of delayed fluorescence responds to inter-sublevel population transfer within triplet $x$-traps, and (b) a decrease in delayed fluorescence intensity accompanies an increase in $\mathrm{x}$-trap phosphorescence intensity for each case where both signals are observed.

An increase in phosphorescence intensity in a slow passage ODMR transition accompanies the transfer of population from a sublevel of lower to one of higher radiative quantum yield. It is reasonable to assume, as is usually the case, that the sublevel with higher quantum yield also decays more rapidly; thus saturating this zero field transition during continuous optical pumping leads to an increase in the phosphorescence intensity and a net depletion of the steady state $T_{1}$ population. Since the triplet exciton sublevels remain equally populated through rapid spin-lattice relaxation, heterofusion processes which lead to the production of singlets [40] and therefore delayed fluorescence, should be insensitive to the redistribution of $x$-trap sublevel populations, but they should vary (roughly linearly in our measurements) with the total x-trap population. Thus, decreases in delayed fluorescence intensity which accompany increases in $\mathrm{x}$-trap phosphorescence intensity are readily understood.
The inhomogeneous optical linewidth of the crystal $T_{1} \leftarrow S_{0}$ origins, between $1 \mathrm{~cm}^{-1}$ and $3 \mathrm{~cm}^{-1}$, are determined by the quality of the crystal, which is greatly affected in this case by photochemically-induced trap states. Isotopic sites which could be resolved in some of the spectra have already been reported [17-19], and were not investigated in this work.

The crystal lattice of $s$-tetrazine with factor group $\mathrm{C}_{2 \mathrm{~h}}^{15}$ contains two incquivalent molcculcs in the unit cell [41]. The triplet exciton band should be split into two Davydov components, which we could not resolve (fig. 6a). Analogous results were obtained for 2 and 4, where the inhomogenous linewidth does not allow for the resolution of Davydov splittings which would be expected for 2, at least, on the basis of its crystal structure [42]. The crystal structure of 4 has not been determined. The Davydov splittings of 3 have been discussed previously [31].

\section{Conclusions}

The electronic spectroscopy of $s$-tetrazine (1) and its 3,6-disubstituted analogs (2-4) has been investigated with particular attention to the lowest excited triplet states $\left(T_{1}\right)$ on which ODMR measurements were made. The positions of the $S_{1}$ and $T_{1}$ exciton band origins are reported for 1-4. Delayed fluorescence was observed in each of the four $s$-tetrazine single crystals when pumping the $T_{1} \leftarrow S_{0}$ exciton band origin, but not when pumping a $T_{1} x$-trap origin. The identification of $x$-trap ODMR signals of 1-4 when monitoring delayed fluorescence confirms that this emission originates at least partially from triplettriplet annihilation through an exciton-trap heterofusion process. ODMR signals were observed by monitoring the phosphorescence of 1,3 and 4 , and the $\mathrm{zfs}$ of several traps in $\mathbf{3}$ and $\mathbf{4}$ were assigned. The phosphorescence of 3 and $\mathbf{4}$ could also be induced by optical pumping of $S_{1} \leftarrow S_{0}$, although none was observed in 1 using this mode of excitation. These observations confirm that isc in $s$-tetrazines increases with substitutions at the 3 and 6 positions. The opposite relative signs of the ODMR signals observed via phosphorescence and delayed fluorescence was explained by a simple dynamic model.

Phosphorescence excitation spectra obtained at the 
$T_{1}$ origin reveal inhomogenously broadened bands with widths of $1-3 \mathrm{~cm}^{-1}$, attributed largely to inferior crystal quality resulting from photochemically induced defects. Structure, attributed to Davydov splittings, could be observed only in 3,6-dimethoxy$s$-tetrazine (3).

The observed zfs reveal a nearly axially symmetric fine structure tensor with a large value of $|D|(\approx 0.4$ $\mathrm{cm}^{-1}$ ) consistent with a triplet $\mathrm{n} \pi^{*}$ state. Calculations based on a magnctic dipole-dipolc modcl for the $\mathrm{zfs}$ of $\mathrm{a}^{3} \mathrm{~B}_{3 \mathrm{u}}$ state are in good agreement with the observed $|D|$ value, overestimating it by only $12 \%$. The good agreement of the dipole-dipole interaction model for $s$-tetrazine, in contrast with the significantly poorer agreement for the $n \pi^{*} T_{1}$ state of pyrazine, suggests the more important role of spin-orbit coupling contributions to the $\mathrm{zfs}$ of the latter molecule.

$D$ of s-tetrazine is calculated to be positive, and the principal axis of maximum $\mathrm{zfs}$ is predicted to lie along the direction connecting the 3 and 6 carbon atom positions of the $s$-tetrazine ring.

\section{Acknowledgement}

AHM. would like to thank the WE-Heraeus-Stiftung for a grant which made possible his contributions to this work.

\section{References}

[1] J. Koenigsberger and K. Vogt, Physik. Z. 14 (1913) 1269.

[2] C. Lin, E. Lieber and J. Horwitz, J. Am. Chem. Soc. 76 (1954) 472.

[3] S.F. Mason, J. Chem. Soc. (1959) 1210.

[4] G.H. Spencer, P.C. Cross and K.B. Wiberg, J. Chem. Phys. 35 (1961) 1925.

[5] A.J. Merer and K.K. Innes, Proc. Roy. Soc. A 302 (1968) 271.

[6] R. Gleiter, V. Schehlmann, J. Spanget-Larsen, H. Fischer and F.A. Neugebauer, J. Org. Chem. 53 (1988) 5756.

[7] M. Chowdhury and L. Goodman, J. Chem. Phys. 36 (1962) 548.

[8] D.T. Livak and K.K. Innes, J. Mol. Spectry. 39 (1971) 115.

[9] J.R. McDonald and L.E. Brus, J. Chem. Phys. 59 (1973) 4966.

[10] R.M. Hochstrasser and D.S. King, Chem. Phys. 5 (1974) 439.
[11] R.M. Hochstrasser and C.T. Marzzacco, J. Chem. Phys. 38 (1963) 2979.

[12] G.K. Vemulapalli and T. Cassen, J. Chem. Phys. 56 (1972) 5120.

[13] J. De Vries, D. Bebelaar and J. Langelaar, Opt. Commun. 18 (1976) 24;

J. Langelaar, B. Bebelaar, M.W. Leeuw, J.J.F. Ramaekers and R.P.H. Rettschnick, in: Proceedings of the Second International Conference on Picosecond Phenomena (Springer, Berlin, 1980) p. 147.

[14] C.A. Hayam, L. Young, C. Morter and D.H. Levy, J. Chem. Phys. 81 (1984) 5216.

[15] J.H. Meyling, R.P. van der Verf and D.A. Wiersma, Chem. Phys. Letters 28 (1974) 364.

[16] J.M. Paczkowski, R. Pierce, A.B. Smith III and R.M. Hochstrasser, Chem. Phys. Letters 72 (1980) 5.

[17] R.M. Hochstrasser and D.S. King, J. Am. Chem. Soc. 97 (1975) 4760 .

[18] B. Dellinger, D.S. King, R.M. Hochstrasser and A.B. Smith III, J. Am. Chem. Soc. 99 (1977) 3197.

[19] B. Dellinger, D.S. King, R.M. Hochstrasser and A.B. Smith III, J. Am. Chem. Soc. 99 (1977) 7138.

[20] D.M. Burland, F. Carmona and J. Pacansky, Chem. Phys. Letters 56 (1978) 221.

[21] J.C. Alfano, S.J. Martinez III and D.H. Levy, J. Chem. Phys. 94 (1991) 2475, and references therein.

[22] S. Ghosh and M. Chowdhury, Chem. Phys. Letters 85 (1982) 233.

[23] X. Zhao, W.B. Miller, E.J. Hintsa and Y.T. Lee, J. Chem. Phys. 90 (1989) 5527.

[24] A.C. Scheiner, G.E. Scuseria and H.F. Schaefer III, J. Am. Chem. Soc. 108 (1986) 8160.

[25] H. Sternlicht, J. Chem. Phys. 38 (1963) 2316.

[26] D.L. Boger, R.S. Coleman, J.S. Panek, F.X. Huber and J. Saver, J. Org. Chem. 50 (1985) 5377;

G.H. Spencer Jr., P.C. Cross and K.B. Wilberg, J. Chem. Phys. 35 (1961) 1939;

W. Skorianetz and E. Kovats, Helv. Chim. Acta 54 (1971) 1922;

F.A. Neugebauer and H. Fischer, Liebigs Ann. Chem. (1982) 387;

J. Sandström, Acta Chem. Scand. 15 (1961) 1575.

[27] F. Gückel, D. Schweitzer, J.P. Collman, S. Bencosme, E. Evitt and J. Sessler, Chem. Phys. 86 (1984) 161.

[28] J. Schmidt and J.H. van der Waals, Chem. Phys. Letters 2 (1968) 640 .

[29] R.M. Hochstrasser, D.S. King and A.B. Smith III, J. Am. Chem. Soc. 99 (1977) 3923.

[30] K.W. Benz, Dissertation, University of Stuttgart (1970).

[31] F. Gückel, Dissertation, Ruprecht-Karls-University, Heidelberg (1983);

F. Gückel, D. Schweitzer, K.H. Hausser and F.A. Neugebauer, Abstracts of the Tenth Molecular Crystal Symposium, St. Jovite, Quebec (1982) p. 106.

[32] C. Krieger, H. Fischer, F.A. Neugebauer, F. Gückel and D. Schweitzer, Acta Cryst. C 43 (1987) 1412. 
[33] M.S. de Groot, I.A.M. Hesselmann and J.H. van der Waals, Mol. Phys. 16 (1969) 45.

[34] H. Hayashi and S. Nagakura, Mol. Phys. 24 (1972) 801; Chem. Phys. Letters 18 (1973) 63;

C.R. Jones, A.H. Maki and D.R. Kearns, J. Chem. Phys. 59 (1973) 873.

[35] M. Sharnoff, Chem. Phys. Letters 2 (1968) 498;

R.F. Clements and M. Sharnoff, Chem. Phys. Letters 7 (1970) 4.

[36] L.J. Cheng and A.L. Kwiram, Chem. Phys. Letters 4 (1969) 457.
[37] R.M. Hochstrasser and C. Marzzacco, J. Chem. Phys. 49 (1968) 971.

[38] H. Vogler, Z. Naturforsch. 41 a (1986) 959.

[39] R.M. Hochstrasser and T.-S Lin, J. Chem. Phys. 53 (1970) 2676.

[40] R.E. Merrifield, J. Chem. Phys. 48 (1968) 4381.

[41] F. Berntinotti, G. Giacomello and A.M. Liquori, Acta Cryst. 8 (1955) $513 ; 9$ (1956) 510.

[42] J.C. Huffmann, Cryst. Struct. C 10 (1981) 227. 